\title{
Aberration-Corrected STEM and Atomic EELS Imaging Study of Defects and Interfaces in Thin Films of Layered Structures
}

\author{
N. Gauquelin*, M. Couillard*, H. Zhang**, J. Y.T. Wei *****, G.A. Botton* \\ * Canadian Centre for Electron Microscopy and Department of Materials Science and Engineering, \\ McMaster University, 1280 Main Street West, Hamilton, ON, L8S 4M1, Canada \\ ** Department of Physics, University of Toronto, 60 St. George St., Toronto, ON M5S1A7, Canada \\ *** Canadian Institute for Advanced Research, Toronto, M5G1Z8, Canada
}

Layered compounds are extensively studied for applications as superconductors and ionic conductors. These layered structures can be considered as stacking sequences of perovskite layers with or without rock-salt layers separating them and where planar defects such as stacking faults are often present. This anisotropy of defects and the structure of interfaces with the substrates have potentially dramatic effects on the physical properties such as low dimensional transport, diffusion and growth. In this study we focus our attention to the interface structure and defects on $\mathrm{YBa}_{2} \mathrm{Cu}_{3} \mathrm{O}_{7-\delta}$ thin films grown on different substrates of SrTiO3 (STO) and $\left(\mathrm{LaAlO}_{3}\right)_{0.3}$ $\left(\mathrm{Sr}_{2} \mathrm{AlTaO}_{6}\right)_{0.7}(\mathrm{LSAT})$ ultimately used for transport measurements in YBCO-LCMO multilayer [1].

Imaging with high-angle annular dark-field scanning transmission electron microscopy (HAADFSTEM) and electron energy loss spectroscopy (EELS) were carried out with an FEI Titan 80-300 Cubed TEM, equipped with a CEOS-designed hexapole-based aberration corrector for the probeforming lens and imaging lens. The instrument is fitted with a high brightness electron source, a Super-Twin lens in order to achieve sub-Ångstrom resolution both for phase contrast imaging, STEM and EELS mapping [2]. Measurements were realized at 80 and $200 \mathrm{keV}$ in order to reduce knock-on damage. EELS spectra were recorded on 2048 channels with an energy dispersion of $0.5 \mathrm{eV}$. EELS spectrum images were refined using Weighted Principal Component Analysis using the Multivariate Statistical Analysis plugin developed by M. Watanabe [3] and implemented by "HREM Research" within Gatan's DigitalMicrograph. The effects of the MSA on the data is discussed elsewhere [4].

EELS mapping shows that the STO last layer at interface between STO and $\mathrm{YBa}_{2} \mathrm{Cu}_{3} \mathrm{O}_{7-\delta}$ (YBCO123) terminated by $\mathrm{Ti}$ atoms and that the first layer is $\mathrm{Ba}-\mathrm{O}$ (figure 1). Also from direct atomic EELS imaging, we note that, after the first YBCO unit cell, we detect a shift of the Ba atomic positions suggesting that, in the analyzed area, the $\mathrm{YBa}_{2} \mathrm{Cu}_{4} \mathrm{O}_{8}(\mathrm{YBCO} 124)$ cell appears. Such complex stacking is exemplified in the growth of YBCO on $\left(\mathrm{LaAlO}_{3}\right)_{0.3}\left(\mathrm{Sr}_{2} \mathrm{AlTaO}_{6}\right)_{0.7}$ (LSAT) (figure 2). From the combination of atomic resolved maps and HAADF intensities, we can unambiguously identify the sequence of layers and intergrowths of $\mathrm{Cu}-\mathrm{O}_{2}$ layers. From these images, we identified the local fraction of the YBCO124 phase and the role of this phase on the nature of the interface between YBCO and LCMO ultrathin films. It was found that interfaces have a different layer arrangement on the first few nanometers of the film and that films can present a high fraction of YBCO124 in some growth conditions. Our results also demonstrate that smooth interfaces, at the unit cell level and over several microns, can be obtained and thus very high epitaxial quality of the samples can be grown. 
References

[1] I. Friedman et al., Physical Review B, (2010) submitted

[2] Botton, G.A., Lazar, S., Dwyer, C., ULTRAMICROSCOPY, 110, 926-934(2010)

[3] M. Watanabe et al., Proc. Inter. Conf. on Solid-Solid Phase Transformations in Inorganic Materials 2005 - Vol. 2, pp. 431-442 (2005)

[4] Dudeck et al., Submitted to Micron.

[5] We are grateful to NSERC (Discovery grant) and CIFAR for supporting this work.
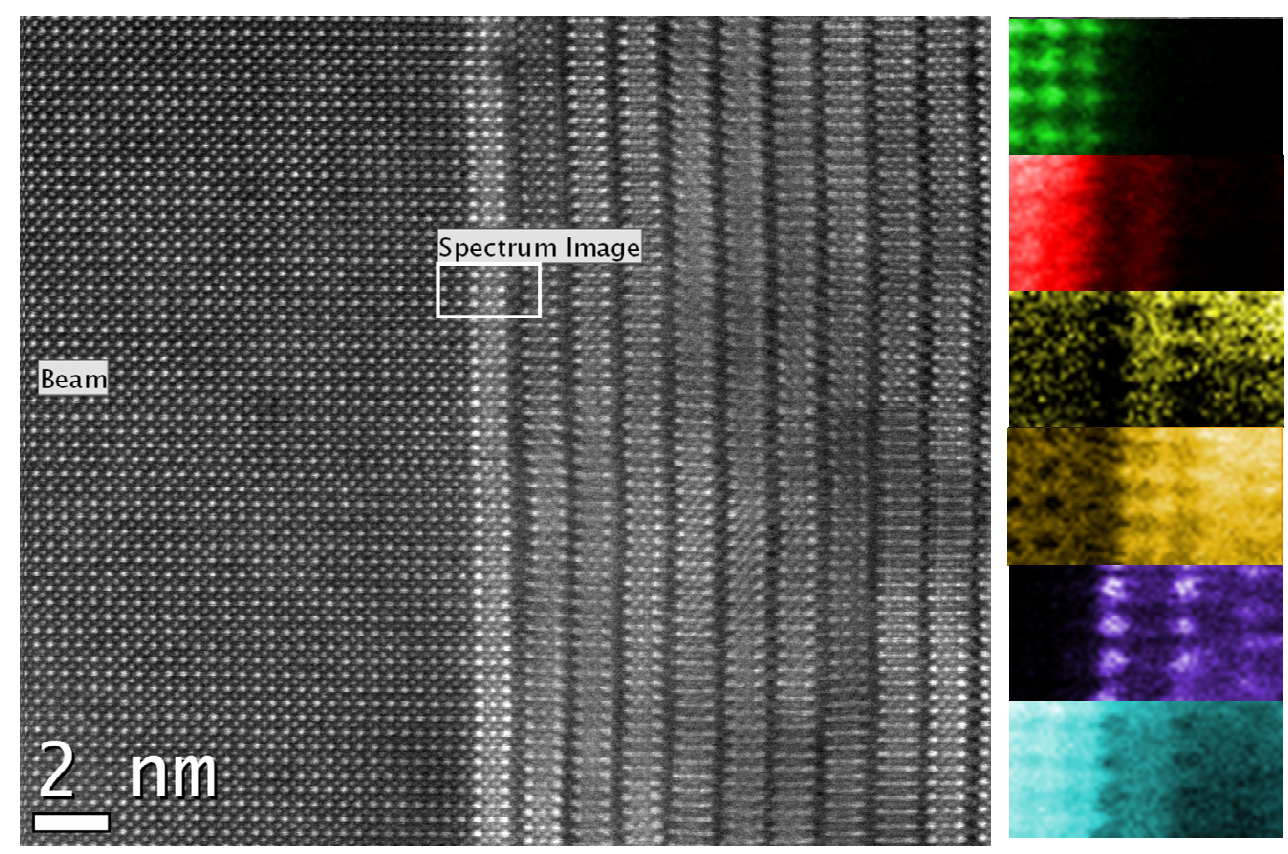

Ti L edge

Sr $M$ edge

Y M edge

Cu $\mathrm{L}$ edge

Ba $M$ edge

$O K$ edge

FIG. 1. HAADF STEM image with corresponding EELS mapping of the different elements of the interface of a YBCO (100) thin film grown on STO (100) substrate showing the atomic termination of the interface (one layer of $\mathrm{TiO}$ followed by $1 \mathrm{BaO}$ layer) as well as the different relative concentrations of oxygen in the substrate as well as in the $\mathrm{BaO}, \mathrm{CuO}$ and $\mathrm{CuO}_{2}$ layers of $\mathrm{YBCO}$.

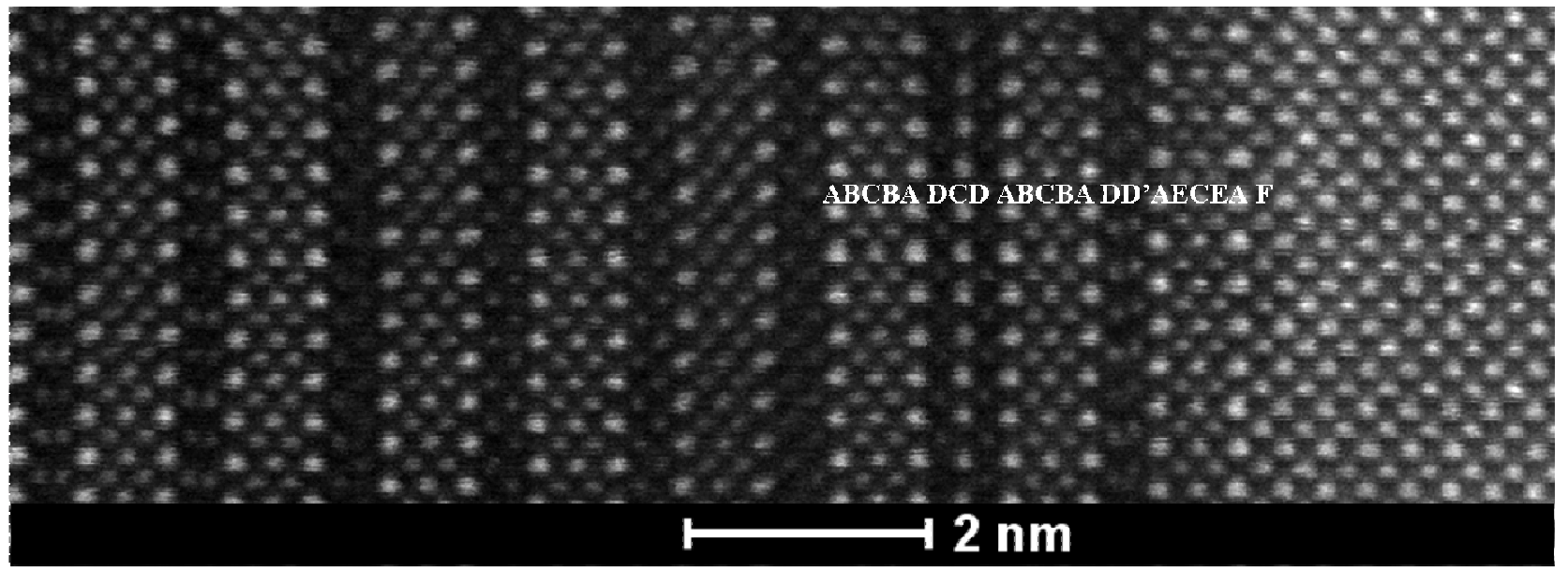

FIG. 2. a) HAADF STEM image of the LSAT/YBCO interface showing that the main phase is YBCO124. b) Detail of the interface of a YBCO (100) thin film grown on LSAT (100) substrate showing the appearance of a different phase at the interface. $\mathrm{A}: \mathrm{BaO}, \mathrm{B}: \mathrm{CuO}_{2}, \mathrm{C}: \mathrm{Y}, \mathrm{D}: \mathrm{CuO}$, D’: CuO(shifted), E:La(Sr)O, F:Al(Ta)O. 